学 位 論 文

Single-nucleotide polymorphisms in the human $R A D 21 L$ gene may be a genetic risk factor for Japanese patients with azoospermia caused by meiotic arrest and Sertoli cell-only syndrome

（減数分裂停止およびSertoli cel1-only syndromeに起因する 日本人無精子症患者におけるRAD21L遺伝子の 解析に関する研究)

旭川医科大学大学院医学系研究科博士課程 臨床生殖・発達・再生医学領域専攻

水無瀨 学

（宮本敏伸，宮川康，飯島将司，上田寛人， 西條泰明，並木幹夫，千石一雄） 


\title{
Single-nucleotide polymorphisms in the human RAD21L gene may be a genetic risk factor for Japanese patients with azoospermia caused by meiotic arrest and Sertoli cell-only syndrome
}

\author{
Gaku Minase $^{\mathrm{a}}$, Toshinobu Miyamoto ${ }^{\mathrm{a}}$, Yasushi Miyagawa ${ }^{\mathrm{b}}$, Masashi lijima ${ }^{c}$, Hiroto Ueda ${ }^{\mathrm{a}}$, Yasuaki Saijo $^{\mathrm{d}}$, \\ Mikio Namiki ${ }^{c}$ and Kazuo Sengoku ${ }^{a}$

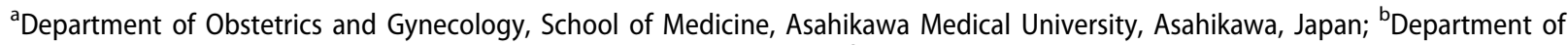 \\ Urology, Osaka University Graduate School of Medicine, Suita, Japan; 'Department of Integrated Cancer Therapy and Urology, \\ Kanazawa University Graduate School of Medical Science, Kanazawa, Japan; dDivision of Community Medicine and Epidemiology, \\ Department of Health Science, School of Medicine, Asahikawa Medical University, Asahikawa, Japan
}

ABSTRACT

Genetic mechanisms are implicated in some cases of male infertility. Recently, it was demonstrated that male mice lacking the gene for RAD21L exhibited azoospermia caused by meiotic arrest. Mouse $R A D 21 L$ is a functionally relevant meiotic $\alpha$-kleisin that is essential for male fertility. Therefore, we hypothesized that $R A D 21 L$ mutations or polymorphisms may be associated with male infertility, especially azoospermia secondary to meiotic arrest. To determine if RAD21L defects are associated with azoospermia in groups of patients with meiotic arrest, we performed direct sequencing of the RAD21L coding regions in 38 Japanese patients with meiotic arrest and in 200 normal controls. Three coding single-nucleotide polymorphisms (SNP1-SNP3) were detected in the meiotic arrest patient group. Sertoli cell-only syndrome is considered a common cause of non-obstructive azoospermia. For comparison, the RAD21L coding regions in which SNP1-SNP3 were detected were sequenced in 140 patients with Sertoli cell-only syndrome. Statistical analyses were used to compare the two groups of patients with the control group. Genotype and allele frequencies of SNP2 and SNP3 were notably higher in the two patient groups compared with the control group (Bonferroni adjusted $p$ value $<0.016$ ). These results suggest a critical role for $R A D 21 L$ in human spermatogenesis.
ARTICLE HISTORY

Received 29 February 2016

Accepted 7 July 2016

KEYWORDS

Azoospermia; meiotic arrest; RAD21L; SCOS; single-

nucleotide polymorphism

\section{Introduction}

Infertility affects $\sim 15 \%$ of couples trying to conceive, and approximately half of these cases involve male factors (Krausz, Escamilla, \& Chianese, 2015). Genetic causes of azoospermia include chromosomal abnormalities, $Y$ chromosome microdeletions and specific mutations/deletions of several $\mathrm{Y}$ chromosomal genes (Miyamoto, Minase, Okabe, Ueda, \& Sengoku, 2015). Approximately $10 \%$ of all male infertility cases $(<1 \%$ of all men) are associated with non-obstructive azoospermia (Anniballo et al., 2000; Jarow, Espeland, \& Lipshultz, 1989; Vloeberghs et al., 2015). This is a histopathological diagnosis based on testicular biopsy findings including hypospermatogenesis, maturation arrest [also called meiotic arrest (MA)] or Sertoli cell-only syndrome (SCOS). Defective meiosis during spermatogenesis causes azoospermia; however, the mechanisms leading to defective meiosis remain unknown.
Meiosis is obviously a fundamental process in sexually reproducing species that allows genetic exchange between maternal and paternal genomes (Nasmyth, 2002). Mutations in the RNA-binding motif protein gene $R B M Y(\mathrm{Yq} 11.223)$ and the synaptonemal complex protein gene SYCP3 (12q23) are known to cause azoospermia secondary to MA (Elliott et al., 1997; Matzuk \& Lamb, 2008; Miyamoto et al., 2003). Genetic polymorphisms may also increase susceptibility to some forms of male infertility including azoospermia via MA (Barda et al., 2014; Eggers et al., 2015; Lu et al., 2013).

The cohesion subunit RAD21 is a member of the $\alpha$-kleisin family of proteins, and is present from fish to mammals (Gutiérrez-Caballero et al., 2011; Ishiguro, Kim, Fujiyama-Nakamura, Kato, \& Watanabe, 2011; Lee \& Hirano, 2011). A RAD21 paralogue (RAD21L) is abundantly transcribed in the testis, and is suggested to be a canonical cohesion subunit. RAD21L interacts with 
the structural maintenance of chromosomes proteins SMC3 and SMC $1 \alpha / \beta$, and with the stromal antigen STAG3 (Gutiérrez-Caballero et al., 2011; Ishiguro et al., 2011; Lee \& Hirano, 2011). Moreover, RAD21L localizes along the axial elements of the synaptonemal complex of mouse meiocytes (Herrán et al., 2011). Male mice lacking RAD21L are defective in the full synapsis of homologous chromosomes at the zygotene stage, which leads to total azoospermia and consequently infertility (Herrán et al., 2011).

To determine if $R A D 21 L$ plays a critical role in human spermatogenesis, we performed mutational analysis of the human RAD21L gene using genomic DNA from Japanese patients with azoospermia caused by MA and SCOS.

\section{Materials and methods}

\section{Patients and controls}

This study was approved by the Ethics Committee of Asahikawa Medical University, Japan. Written informed consent for this study was obtained from each participant. Patients with azoospermia secondary to MA without any chromosomal abnormalities were selected and histopathologically examined what material was examined on all of the patients with azoospermia. A total of 38 Japanese patients with azoospermia due to MA and 140 patients with azoospermia caused by SCOS were included in the study. Two hundred fertile men who had produced at least one child and lacked any history of infertility treatment were included as normal controls. All participants were Japanese.

\section{Mutation screening}

Peripheral blood leukocytes were obtained from 38 and 140 patients with MA and SCOS, respectively, and 200 normal controls. Genomic DNA was extracted using a Wizard ${ }^{\mathrm{TM}}$ Genomic DNA Purification Kit (Promega, Madison, WI) according to the manufacturer's instructions. The RAD21L CDNA sequence (HQ603827.1) was compared with the human genomic sequence (NC_018931) by BLAST, and all exon-intron borders were determined. The PCR primer sets used to amplify the coding regions are listed in Table 1 . Nested PCRs were performed using the DNAs from the MA patients and the normal controls as templates. The PCR products were purified using a QIAquick PCR Purification kit (Qiagen, Tokyo, Japan). Direct sequencing of each product was performed. The DNAs from the SCOS patients were sequenced using the same procedure, but only in the three regions in which SNPs were detected.

\section{Genotyping and statistical analysis}

Fisher's exact test was used to evaluate the statistical significance of RAD21L variants in the azoospermia patients. Bonferroni post hoc corrections were applied to correct for comparisons among the three RAD21L variants discovered (adjusted $p<0.016$ ). Hardy-Weinberg equilibrium (HWE) was tested for the variants using SNPAlyze software (Dynacom, Chiba, Japan). Linkage disequilibrium of all possible two-way SNP combinations was tested by calculating absolute correlation coefficient values. Haplotype frequencies

Table 1. Oligonucleotide primer sequences used for mutational screening of RAD21L.

\begin{tabular}{|c|c|}
\hline Forward primers & Reverse primers \\
\hline Exon 1 F1: 5'-TGTTAGTAAGAAGAGAACTCAAATC-3' & R1: 5'-GAATAAGAAAGGAATATGAATACGC-3' \\
\hline F2: $5^{\prime}$-GGTTAAGAAAGGAATATGAATACGC- $3^{\prime}$ & R2: 5'-TAAAGGAAAGACCATTTGACAAGTA-3' \\
\hline Exon 2 F1: 5'-CTGAACCAAGACCATTTGACAAGTA-3' & R1: $5^{\prime}$-GGCACGACAAACTAAATGACCCA-3' \\
\hline F2: 5'-ACTACAGAGTTTATATTCCTCTCAT-3' & R2: $5^{\prime}$-GCAGGTATAGAAAAAGAGCAAGG-3' \\
\hline Exon 3 F1: $5^{\prime}$-GTATGGTCAGTATGGTGAATGTC- $3^{\prime}$ & R1: 5'-TATCTCCTCAGTGCCTAGAACAG-3' \\
\hline F2: $5^{\prime}$-CTGCTTTATAGAGAATGGACTACAC- $3^{\prime}$ & R2: 5'-CACACTTTTGTTGTTGTACTTTGGG-3' \\
\hline Exon 4 F1: $5^{\prime}$-CTCAACATCTAAGTCAAGTGCTG-3' & R1: 5'-TCTCTCACACCCAGAATCTCCTT- $3^{\prime}$ \\
\hline F2: 5'-CACTGGATTTGGAAGCCTAGAGA-3' & R2: $5^{\prime}$-CCTGTCTATTGATAGCACCTGATA-3' \\
\hline Exon 5 F1: $5^{\prime}$-TGTTCCTTATTTTCCAAACCTCCAC-3' & R1: 5'-AATATACTAACCTCACTCTCTCCC-3' \\
\hline F2: 5'-AGTCTTTCAGCCATTCTCTTCATC-3' & R2: 5'-AACCTCACTCTCTCCCCTCCAAA-3' \\
\hline Exon 6 F1: 5'-AAAGATTATTTATTGGCTGTTCC-3' & R1: 5'-TCTACTGTTTTTAGAAAGCA-3' \\
\hline F2: $5^{\prime}$-TTATTTATTGGCTGTTCCTATCA-3' & R2: 5'-CCTATTTTATATTGTCTACAGTC- $3^{\prime}$ \\
\hline Exon 7 F1: 5'-AGTCATTTACTGTAGTTGCTGCC- $3^{\prime}$ & R1: $5^{\prime}$-AGTAGGAATCATTGGGTCATCAC-3' \\
\hline F2: $5^{\prime}$-CATATAATCTGTAAGCCTCTTCAGC-3' & R2: 5'-CCGTCTTCTCCTCTATTGTGTTAC-3' \\
\hline Exons 8, 9 F1: 5'-ACTGTAGGTTGTACTGTGGTAG-3' & R1: 5'-TTTGAGTATGCCTTTGGGAGTG-3' \\
\hline F2: 5'-AGTGTAGTGATGTGGTCTCAGC-3' & R2: $5^{\prime}$-GAGTGAAGGAGGTAGAAAGAGTG-3' \\
\hline Exon 10 F1: $5^{\prime}$-TTTCTGTCTACCCAAAAGGCACAT- $3^{\prime}$ & R1: 5'-GTAGGATTCTCAGCATATAACTAGG-3' \\
\hline F2: 5'-AATGTGTGAGAGCGTGTGTAGAG-3' & R2: $5^{\prime}$-GGTATGAAACCACCTTATCTTGC- $3^{\prime}$ \\
\hline Exon $11 \mathrm{~F} 1: 5^{\prime}$-CAGTAGCACAGCAGTAGAGACTT- $3^{\prime}$ & R1: 5'-TTGTTCAGATTTCCAGAAGCCTTG-3' \\
\hline F2: 5'-GTTCTTCCTTTATGGGAGGCATAT-3' & R2: 5'-CCTGTCACTACTTTTGATAAGCACA- $3^{\prime}$ \\
\hline Exon 12 F1: $5^{\prime}$-AAAGTAACCGTAAGACCCTGGACA-3' & R1: 5'-GATAAGGAGAGGTAGGACAAAGG-3' \\
\hline F2: 5'-CTATTTGGAGCCTCTTATCAGAAG-3' & R2: 5'-ATCTAAGGGTATTAGTGTGGGTAAG-3' \\
\hline Exon 13 F1: $5^{\prime}$-GTGTTGTTTTAATGTTAACTAAGG-3' & R1: 5'-TAAGTATTTCTGAGCCAGGTATG-3' \\
\hline F2: 5'-GAGTCATTTCCCTTTTTCCATTTA-3' & R2: $5^{\prime}$-CAATATAGTGAGACCTTTGTCCTT-3' \\
\hline
\end{tabular}


were estimated by the maximum likelihood method based on the expectation-maximization algorithm under the assumption of HWE. Linkage disequilibrium and haplotype frequency were tested using SNPAlyze software. All $p$ values were determined by $\chi^{2}$ approximation, with significance considered at $p$ values $<0.05$.

\section{Results}

The RAD21L coding region was sequenced in $38 \mathrm{MA}$ patients. No apparent mutations were found, but three variants were detected in the patient group compared with the control group (Table 2). The detected variants were: c.454C > A (SNP1) (chr20: 1234085) in exon 4; c1268A > C (SNP2) (chr20: 1243196) in exon 10 and c1610G > A (SNP 3) (chr20: 1254311) in exon 14. Only SNP1 has been reported previously (rs755285899); however, we were unable to find information on its frequency in the Japanese population. The allele and genotype distributions of the three SNPs in both MA patients and controls are shown in Table 2. Allele and genotype distributions adhered to HWE $(p>0.05)$. Association was observed for SNP2 $(p<0.016)$ and SNP3 $(p<0.016)$, but not for SNP1 (Table 2). For comparison, 140 patients with azoospermia caused by SCOS were also analysed but only in the coding regions containing SNP1-SNP3. The frequencies of SNP2 and SNP3 were also higher in the SCOS patients compared with the control group $(p<0.016)$.

Haplotype analysis revealed similar estimated haplotype frequencies for all three SNPs $(p>0.05)$. Haplotype estimation and linkage disequilibrium analysis also revealed no critical differences $(p>0.05)$. In addition, SNP2 and SNP3 were detected in different individuals in both patient groups.

\section{Discussion}

In this study, we hypothesized that mutations or polymorphisms in RAD21L may be associated with azoospermia caused by $\mathrm{MA}$ and SCOS. No RAD21L mutations that directly cause azoospermia were detected; however, the small numbers of patients analysed, especially those with MA, was not enough to reach an absolute conclusion. Nevertheless, we did identify three SNPs within the RAD21L coding regions. Moreover, we found that the distribution of the SNP2 [1268A $>$ C (His423Pro)] and SNP3 [1610G $>$ A (Ser537Asn)] genotypes was significantly different between Japanese azoospermia patients with MA or SCOS, and fertile controls $(p<0.016)$. This indicates that the $C$ allele of SNP2 (in exon 10) and the resulting Pro amino acid substitution, and the A allele of SNP3 (in exon 14) and the resulting Asn amino acid substitution, or their flanking regions, may play a role in the disruption of spermatogenesis in Japanese patients. Again, the numbers of patients analysed were not large enough to allow a definitive conclusion to be drawn. The function of the SNPs at positions 1268 and 1610 in the gene sequence is unknown; however, the SNP2 and SNP3 changes were predicted to be 'benign' in an in silico analysis using PolyPhen2 and MutationTaster. Azoospermia with MA and SCOS is very rare, and our histological diagnostic criteria were very strict. Indeed, we have DNA samples from more than 5000 patients with azoospermia, and only 38 and 140 of them had azoospermia caused by MA and SCOS. Among the others, 15 and 1286 cases were for hypospermatogenesis and obstructive azoospermia, respectively. For the remaining patients, we could not determine if they had obstructive or non-obstructive

Table 2. Genotype and allele frequencies for three coding SNPs in the human RAD21L gene identified in 38 azoospermia patients with meiotic arrest, 140 with SCOS and 200 normal controls.

\begin{tabular}{|c|c|c|c|c|c|c|c|c|}
\hline \multirow[b]{4}{*}{ SNP } & & & \multicolumn{3}{|c|}{ Genotype frequency } & \multicolumn{3}{|c|}{ Allele frequency } \\
\hline & & & \multicolumn{3}{|c|}{ Genotype/total no. of samples (\%) } & \multicolumn{3}{|c|}{ Minor allele/total no. of chromosomes (\%) } \\
\hline & \multicolumn{2}{|c|}{ Alteration } & \multicolumn{3}{|c|}{$p$ value $^{\mathrm{b}}$} & \multicolumn{3}{|c|}{$p$ value $^{\mathrm{b}}$} \\
\hline & Nucleotide & Amino acid & (G) $M A^{a}$ & $\mathrm{~s} \cos ^{\mathrm{c}}$ & Control & (A) $M A^{a}$ & $\mathrm{scos}^{\mathrm{c}}$ & Control \\
\hline $\mathrm{SNP}^{\mathrm{d}}$ & $454 C>A$ & NS & (CA) $12 / 38(31.6)$ & $46 / 140(32.9)$ & $53 R / 200(26.5)$ & $\begin{array}{c}\text { (A) } 58 / 76(76.3) \\
0.7749\end{array}$ & $\begin{array}{c}222 / 280(79.3) \\
0.1427\end{array}$ & $297 / 400(74.3)$ \\
\hline & & & $\begin{array}{c}\text { (AA) } 23 / 38(60.5) \\
0.7289\end{array}$ & $\begin{array}{c}88 / 140(62.9) \\
0.0226\end{array}$ & $122 / 200(61.0)$ & & & \\
\hline SNP2 & $1268 \mathrm{~A}>\mathrm{C}$ & NS & $\begin{array}{c}\text { (AC) } 4 / 38(10.5) \\
0.007^{*}\end{array}$ & $\begin{array}{c}10 / 140(7.14) \\
0.005^{*}\end{array}$ & $2 / 200(1.00)$ & $\begin{array}{c}\text { (A) } 4 / 76(5.26) \\
0.007^{*}\end{array}$ & $\begin{array}{c}10 / 280(3.57) \\
0.005^{*}\end{array}$ & $2 / 400(0.50)$ \\
\hline SNP3 & $1610 \mathrm{G}>\mathrm{A}$ & NS & $\begin{array}{c}\text { (GA) } 3 / 38(7.89) \\
0.004^{*}\end{array}$ & $\begin{array}{c}6 / 140(4.29) \\
0.005^{*}\end{array}$ & $0 / 200(0.00)$ & $\begin{array}{l}\text { (A) } 3 / 76(3.95) \\
0.004^{*}\end{array}$ & $\begin{array}{c}6 \mathrm{l} / 280(2.14) \\
0.005^{*}\end{array}$ & $0 / 400(0.00)$ \\
\hline
\end{tabular}

\footnotetext{
${ }^{\mathrm{a}}$ Meiotic arrest.

${ }^{\mathrm{b}}$ The $p$ values are for the patient group compared with the control group.

'Sertoli cell-only syndrome.

${ }^{\mathrm{d}} \mathrm{SNP1}$ is known SNP rs755285899.

NS, non-synonymous substitution.

$*_{p} \leq 0.016$.
} 
azoospermia because most were too old when we carried out the microdissection testicular sperm extraction (MD-TESE) procedure. Further, some patients did not agree to have MD-TESE or histological biopsy.

In conclusion, we suggest that the $C$ and $A$ variants of the SNPs at positions 1268 and 1610 might be associated with azoospermia secondary to MA and SCOS in the human, but that the relationship between the identified SNPs and the mechanistic cause of azoospermia has not yet been determined. Our results provide insight into the molecular basis of MA and SCOS as possible causes of nonobstructive azoospermia. It remains to be determined whether an association between these variants and azoospermia secondary to MA and SCOS exists in similar patients from other ethnic groups.

\section{Acknowledgements}

This work was supported by Grants-in-Aid from the Suhara Memorial Foundation; Scientific Research (Nos. 25462547, 26462469 and 24249019) from the Japan Society for the Promotion of Science; grants from the Ministry of Health, Labour and Welfare, Japan; the Strategic Research Program for Brain Sciences (No. 11105137) and by a Grant-in-Aid for Scientific Research on Innovative Areas (Transcription Cycle) from the Ministry of Education, Culture, Sports, Science, and Technology of Japan (No. 12024421).

\section{Disclosure statement}

The authors report no conflicts of interest. The authors alone are responsible for the content and writing of this article.

\section{Funding}

This work was supported by Grants-in-Aid from the Suhara Memorial Foundation; Scientific Research [Nos. 25462547, 26462469 and 24249019] from the Japan Society for the Promotion of Science; grants from the Ministry of Health, Labour and Welfare, Japan; the Strategic Research Program for Brain Sciences [No. 11105137] and by a Grant-in-Aid for Scientific Research on Innovative Areas (Transcription Cycle) from the Ministry of Education, Culture, Sports, Science, and Technology of Japan [No. 12024421].

\section{References}

Anniballo, R., Ubaldi, F., Cobellis, L., Sorrentino, M., Rienzi, L., Greco, E., \& Tesarik, J. (2000). Criteria predicting the absence of spermatozoa in the Sertoli cell-only syndrome can be used to improve success rates of sperm retrieval. Human Reproduction, 15, 2269-2277. doi: 10.1093/humrep/ 15.11.2269.

Barda, S., Yogev, L., Paz, G., Yavetz, H., Lehavi, O., Hauser, R., ... Kleiman, S.E. (2014). BRDT gene sequence in human testicular pathologies and the implication of its single nucleotide polymorphism (rs3088232) on fertility. Andrology, 2, 641-647. doi: 10.1111/j.2047-2927.2014.00230.x.
Eggers, S., DeBoer, K.D., van den Bergen, J., Gordon, L., White, S.J., Jamsai, D., ... O'bryan, M.K. (2015). Copy number variation associated with meiotic arrest in idiopathic male infertility. Fertility and Sterility, 103, 214-219. doi: 10.1016/j.fertnstert.2014.09.030.

Elliott, D.J., Millar, M.R., Oghene, K., Ross, A., Kiesewetter, F., Pryor, J., ... Cooke, H. (1997). Expression of RBM in the nuclei of human germ cells is dependent on a critical region of the $Y$ chromosome long arm. Proceedings of the National Academy of Sciences USA, 94, 3848-3853. Retrieved from http://www.pnas.org/content/94/8/3848.abstract.

Gutiérrez-Caballero, C., Herrán, Y., Sánchez-Martín, M., Suja, J.A., Barbero, J.L., Llano, E., \& Pendás, A.M. (2011). Identification and molecular characterization of the mammalian $\alpha$-kleisin RAD21L. Cell Cycle, 10, 1477-1487. doi: 10.4161/cc.10.9.15515.

Herrán, Y., Gutiérrez-Caballero, C., Sánchez-Martín, M., Hernández, T., Viera, A., Barbero, J.L., ... Pendás, A.M. (2011). The cohesin subunit RAD21L functions in meiotic synapsis and exhibits sexual dimorphism in fertility. The EMBO Journal, 30, 3091-3105. doi: 10.1038/emboj.2011.222.

Ishiguro, K., Kim, J., Fujiyama-Nakamura, S., Kato, S., \& Watanabe, Y. (2011). A new meiosis-specific cohesin complex implicated in the cohesin code for homologous pairing. EMBO Reports, 12, 267-275. doi: 10.1038/ embor.2011.2.

Jarow, J.P., Espeland, M.A., \& Lipshultz, L.I. (1989). Evaluation of the azoospermic patient. Journal of Urology, 142, 62-65. Retrieved from: https://www.ncbi.nlm.nih.gov/pubmed/ 2499695.

Krausz, C., Escamilla, A.R., \& Chianese, C. (2015). Genetics of male infertility: From research to clinic. Reproduction, 150, R159-R174. doi: 10.1530/REP-15-0261.

Lee, J., \& Hirano, T. (2011). RAD21L, a novel cohesin subunit implicated in linking homologous chromosomes in mammalian meiosis. Journal of Cell Biology, 192, 263-276. doi: 10.1083/jcb.201008005.

Lu, C., Xu, M., Wang, Y., Qu, Y., Du, G., Wu, W., ... Wang, X. (2013). Genetic variants in meiotic program initiation pathway genes are associated with spermatogenic impairment in a Han Chinese population. PLoS One, 8, e53443. Retrieved from: 10.1371/journal.pone.0053443.

Matzuk, M.M., \& Lamb, D.J. (2008). The biology of infertility: Research advances and clinical challenges. Nature Medicine, 14, 1197-1213. doi: 10.1038/nm.f.1895.

Miyamoto, T., Hasuike, S., Yogev, L., Maduro, M.R., Ishikawa, M., Westphal, H., \& Lamb, D.J. (2003). Azoospermia in patients heterozygous for a mutation in SYCP3. Lancet, 362, 1714-1719. doi: 10.1016/S0140-6736(03)14845-3.

Miyamoto, T., Minase, G., Okabe, K., Ueda, H., \& Sengoku, K. (2015). Male infertility and its genetic causes. Journal of Obstetrics and Gynaecology Research, 41, 1501-1505. doi: 10.1111/jog.12765.

Nasmyth, K. (2002). Segregating sister genomes: the molecular biology of chromosome separation. Science, 297, 559-565. doi: 10.1126/science.1074757.

Vloeberghs, V., Verheyen, G., Haentjens, P., Goossens, A., Polyzos, N.P., \& Tournaye, H. (2015). How successful is TESE-ICSI in couples with non-obstructive azoospermia? Human Reproduction, 30, 1790-1796. doi: 10.1093/humrep/ dev139. 